Asthma bronchiale

\section{Welche Step-up-Therapie für Kinder?}

Lemanske RF Jr et al. Step-up therapy for children with uncontrolled asthma receiving inhaled corticosteroids. N Engl J Med 2010;362:975-985

\section{Hintergrund und Fragestellung}

Die Evidenz zur Step-up-Therapie bei Kindern mit unkontrolliertem Asthma fehlte bislang. In der Studie des Childhood Asthma Research and Education (CARE) Network wurden drei verschiedene Step-up-Regimes miteinander verglichen.

\section{Methodik}

In die Studie wurden 182 Kinder im Alter von 6 bis 17 Jahren aufgenommen, die an unkontrolliertem Asthma litten und mit Fluticason $(2 \times 100 \mu \mathrm{g} / \mathrm{d})$ behandelt wurden. Im Rahmen der Studie erhielten sie randomisiert und verblindet drei Arten Wochen in zufälliger Reihenfolge bei einem Dreifach-Cross-over-Design:

- Fluticason 3 x $250 \mu \mathrm{g} / \mathrm{d}$ (ICS-Stepup),

- Fluticason $100 \mu \mathrm{g} / \mathrm{d}+$ lang wirksamer Beta-2-Adrenozeptor-Agonist 2 x 50 $\mu \mathrm{g} / \mathrm{d}$ (LABA-Step-up), einer Step-up-Therapie über jeweils 16
_ Fluticason 2 x $100 \mu \mathrm{g} / \mathrm{d}+$ Leukotrienrezeptorantagonist 5 oder $10 \mathrm{mg} / \mathrm{d}$ (LTRA-Step-up).

Es wurde eine Kombination von drei klinischen Parametern (Exazerbationen, Tage mit Asthmakontrolle, exspiratorische Einsekundenkapazität) verwendet, um festzustellen, ob die Häufigkeit eines differenziellen Ansprechens auf die Step-upRegimes mehr als $25 \%$ betrug. Als differenzielles Ansprechen wurde bezeichnet, wenn diese Parameter bei einem Patienten in mindestens einer Behandlungsperiode besser waren als in einer anderen.

\section{Ergebnisse}

Die LABA-Step-up-Therapie hatte eine größere Wahrscheinlichkeit, das beste Ansprechen zu erzielen, als die LTRA-Stepup-Therapie (relative Wahrscheinlichkeit 1,6; 95\%-Konfidenzintervall [KI] 1,1-2,3; $\mathrm{p}=0,004)$ und die ICS-Step-up-Therapie (relative Wahrscheinlichkeit 1,7; 95\%-KI 1,2-2,4; $\mathrm{p}=0,002$; Abb. 1).

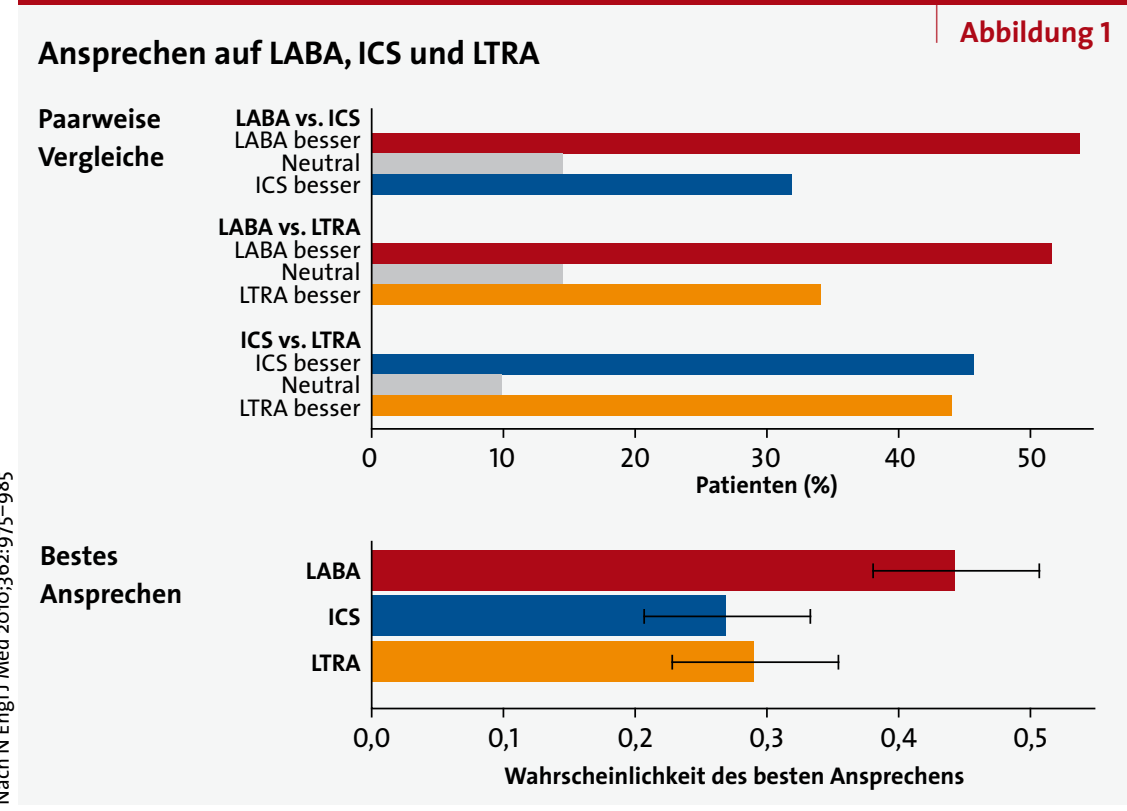

Eine höhere Punktzahl beim Asthmakontrolltest vor der Randomisierung (d.h. eine bessere Asthmakontrolle zu Studienbeginn) war mit einem besseren Ansprechen auf die LABA-Step-up-Therapie verbunden.

\section{Schlussfolgerungen}

Bei fast allen Kindern wurde ein differenzielles Ansprechen auf jede Step-upTherapie gefunden. Die LABA-Step-upTherapie hatte eine signifikant höhere Wahrscheinlichkeit des besten Ansprechens als die ICS- und die LTRA-Stepup-Therapie. Jedoch sprachen viele Kinder auf eine ICS- oder LTRA-Step-upTherapie am besten an.

Dies unterstreicht die Notwendigkeit eines regelmäßigen Monitorings und einer angemessenen Anpassung der Asthmatherapie bei jedem einzelnen Kind.

\section{Kommentar}

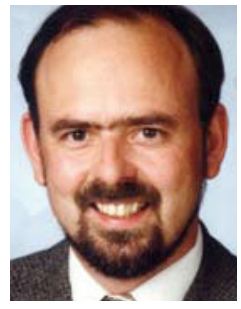

Prof. Dr. med.

Klaus Mörike, Tübingen

Jedes dieser drei Step-up-Verfahren kann bei einem individuellen Patienten wirken. Die Kommentatoren plädieren dafür, die Entscheidung, welchem Verfahren bei einem bestimmten Patienten die erste Wahl zukommt, nach Sicherheit, Preis und Komfort (in dieser Reihenfolge) zu treffen [1].Vor dem Hintergrund immer noch nicht ausgeräumter Sicherheitsbedenken gegenüber LABA in der Asthmabehandlung [2,3] ist ihre erste Wahl entweder die Dosissteigerung von ICS oder die Zugabe eines LTRA zum Therapieregime.

Bei Patienten mit schwer kontrollierbarem Asthma gibt es weiterhin schlichtweg keinen Ersatz für sorgfältiges Followup. Diese Pflicht liegt beim Therapeuten.

\footnotetext{
Literatur

1. Von Mutius E et al. N Engl J Med. 2010;362:10421043

2. Drazen JM et al. N Engl J Med. 2009;360:16711672

3. Chowdhury BA et al. N Engl J Med. 2010;362:11691171
} 